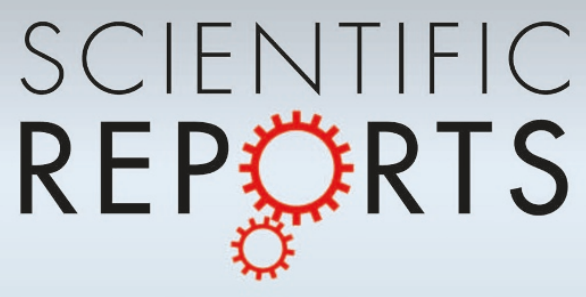

OPEN

SUBJECT AREAS:

QUANTUM HALL

THEORY AND COMPUTATION

SURFACES, INTERFACES AND

THIN FILMS

ELECTRONIC PROPERTIES AND MATERIALS

Received

7 May 2014

Accepted

13 October 2014

Published

19 November 2014

Correspondence and requests for materials should be addressed to

F.L. (fliu@eng.utah. edu)

\section{Formation of quantum spin Hall state on Si surface and energy gap scaling with strength of spin orbit coupling}

\author{
Miao Zhou', Wenmei Ming' ', Zheng Liu', Zhengfei Wang', Yugui Yao² \& Feng Liu',3
}

\begin{abstract}
'Department of Materials Science and Engineering, University of Utah, UT $84112,{ }^{2}$ School of Physics, Beijing Institute of Technology, Beijing, China 100081, ${ }^{3}$ Collaborative Innovation Center of Quantum Matter, Beijing 100084, China.
\end{abstract}

For potential applications in spintronics and quantum computing, it is desirable to place a quantum spin Hall insulator [i.e., a 2D topological insulator (TI)] on a substrate while maintaining a large energy gap. Here, we demonstrate a unique approach to create the large-gap 2D TI state on a semiconductor surface, based on first-principles calculations and effective Hamiltonian analysis. We show that when heavy elements with strong spin orbit coupling (SOC) such as $\mathrm{Bi}$ and $\mathrm{Pb}$ atoms are deposited on a patterned $\mathrm{H}-\mathrm{Si}(111)$ surface into a hexagonal lattice, they exhibit a $2 \mathrm{D}$ TI state with a large energy gap of $\geq 0.5 \mathrm{eV}$. The TI state arises from an intriguing substrate orbital filtering effect that selects a suitable orbital composition around the Fermi level, so that the system can be matched onto a four-band effective model Hamiltonian. Furthermore, it is found that within this model, the SOC gap does not increase monotonically with the increasing strength of SOC. These interesting results may shed new light in future design and fabrication of large-gap topological quantum states.

ecently there has been a surge in the investigation of topological insulators (TIs) ${ }^{1-3}$. TIs are characterized by topologically protected metallic surface or edge states with helical spin polarization residing inside an insulating bulk gap. These states have negligible elastic scattering and Anderson localization ${ }^{4,5}$, which may provide ideal dissipationless spin current for future electronic devices with low power consumption. To realize their potential applications, it is desirable for the TIs to have an energy gap as large as possible ${ }^{6}$, i.e., for room temperature applications. As for 2D TIs [i.e., quantum spin Hall (QSH) insulators], they also need to be grown or placed onto a substrate ${ }^{7-10}$ or formed as an interface ${ }^{12,13}$, while maintaining a large gap. One desired approach is to directly fabricate large-gap TIs on semiconductor surfaces, which may avoid problems like transfer or interfacing a $2 \mathrm{D}$ layer over a foreign substrate ${ }^{11}$. So far, however, this goal remains allusive.

The HgTe quantum well, as the first theoretically predicted ${ }^{12}$ and experimentally confirmed ${ }^{13}$ QSH insulator, has a small gap of $40 \mathrm{meV}$ with topological edge states only detectable at very low temperature $(<10 \mathrm{~K})^{13}$. Recent studies pertaining to $\mathrm{Bi} / \mathrm{Sb}(111)$ films $^{14-17}$, $\mathrm{Sn}$ films ${ }^{18}$, metal-decorated graphen $\mathrm{e}^{19-21}$, silicene/germanene ${ }^{22}$ and $2 \mathrm{D}$ organometallic frameworks ${ }^{23-26}$ have largely enriched the family of $2 \mathrm{D}$ TIs, and some of them have a large gap ${ }^{14,18,21}$. However, a critical drawback with most previous theoretical studies of 2D TIs is their reliance on the electronic and topological properties of freestanding films, whose existence can be in doubt. Even a freestanding film does exist, its properties are expected to be influenced by the underlying substrate in real applications ${ }^{8-10}$.

Here, we demonstrate a unique approach of creating QSH state on a conventional semiconductor surface via depositing heavy elements with strong spin orbit coupling (SOC) onto a patterned Si(111) surface into a hexagonal lattice, which exhibit TI state with a large energy gap of $\sim 0.5 \mathrm{eV}$. Here, the substrate plays a 'positive' role acting as an orbital filter to critically select the orbital composition around the Fermi level to realize nontrivial large-gap topological state ${ }^{27}$. Specifically, the surface system can be matched onto an effective four-band model Hamiltonian which captures the underlying physics. We depict a unified picture of energy gap as a function of SOC to achieve large-gap QSH state. Importantly, we found that it is not necessarily true to have a large gap with a heavier atom of larger SOC, a noteworthy point for future design of TIs.

We have performed density functional theory (DFT) based first-principles calculations of band structure and band topology of $2 \mathrm{D}$ hexagonal lattices of various metal atoms, including $\mathrm{Bi}, \mathrm{Pb}, \mathrm{Sb}, \mathrm{Sn}, \mathrm{Ga}$, In and $\mathrm{Tl}$, grown on a patterned $\mathrm{H}$-saturated $\mathrm{Si}(111)$ surface. The detailed methodologies are presented in the Supplementary Information. We will first discuss in detail the results of $\mathrm{Bi}$ and $\mathrm{Pb}$, as representative examples, and leave the 
results of other metals for later discussion. Atomically flat $\mathrm{H}-\mathrm{Si}(111)$ surface has been prepared for decades and is a widely-used substrate for epitaxial growth of ordered overlayers ${ }^{28,29}$. The surface dangling bonds are passivated by $\mathrm{H}$ to avoid surface reconstruction. In order to form a hexagonal metal overlayer lattice, we propose a two-step fabrication process, as shown in Fig. 1. First, to create a desirable surface template pattern for metal growth, $\mathrm{H}$ atoms are selectively removed in hexagonal symmetry using scanning tunneling microscopy, as discussed in Refs. 30, 31; Second, heavy metal atoms with large SOC can be deposited to grow or self-assemble into the exposed $\mathrm{Si}$ sites, as already demonstrated for other systems $\mathrm{s}^{32-34}$.

We found a very strong binding between the deposited metal atoms and the exposed $\mathrm{Si}$ atoms in the $\mathrm{H}-\mathrm{Si}(111)$ surface, as evidenced by the calculated adsorption length [ $d$, see Fig. 1(c)] of 2.68 $\AA$ and $2.75 \AA$ for $\mathrm{Bi}$ and $\mathrm{Pb}$, respectively. The high structural stability is also indicated by a large adsorption energy $\left(E_{a d}\right)$, defined as $E_{a d}=$ $E_{M} @ H-S i(111)-\left(2 E_{M}+E_{H-S i(111)}\right)+E_{H 2}$, where $E_{M @ H-S i(111)}, E_{M}$, $E_{H-S i(111)}$ and $E_{H_{2}}$ denote the energy of $\mathrm{Bi} / \mathrm{Pb} @ \mathrm{H}-\mathrm{Si}(111)$, single metal atom, pristine $\mathrm{H}-\mathrm{Si}(111)$ surface and $\mathrm{H}_{2}$ gas molecule, respectively. The adsorption energy is found to be $3.88 \mathrm{eV}$ and $3.92 \mathrm{eV}$ for $\mathrm{Bi}$ and $\mathrm{Pb}$, respectively, which are much larger than the binding energies of bulk $\mathrm{Bi}(2.18 \mathrm{eV})$ and $\mathrm{Pb}(2.03 \mathrm{eV})$ in the crystalline solid form, indicating high thermodynamic stability of the surface systems.

To examine the band topology of such surface structures, we first purposely exclude SOC from calculation. The resulting band structures for Bi and $\mathrm{Pb} @ \mathrm{H}-\mathrm{Si}(111)$ are shown in Figs. 2 (a-b), respectively. For Bi@H-Si(111), there are two Dirac bands residing inside the bulk gap of Si with a Dirac point at $K$ point, which locates nearby the Fermi level [Fig. 2(a)]. Analysis of band composition further showed that the two Dirac bands consist mainly of $p_{\mathrm{x}}$ orbitals of $\mathrm{Bi}$ atoms. Another dispersive band, consisting of $p_{\mathrm{y}}$ orbitals of $\mathrm{Bi}$, sits below the bulk conduction band edge of $\mathrm{Si}$ and touches the upper Dirac band of $p_{\mathrm{x}}$ orbitals at $\Gamma$ point. The band structure of $\mathrm{Pb} @ \mathrm{H}-$ $\mathrm{Si}(111)$ is similar to the case of $\mathrm{Bi}$, represented by two Dirac bands inside the Si gap; but the Dirac point sits $\sim 0.8 \mathrm{eV}$ above Fermi level and the upper Dirac band largely overlaps with the conduction band of Si [Fig. 2(b)]. Such different behaviors originate from the different valance electron configuration of $\mathrm{Pb}$ and $\mathrm{Bi}$, e.g., $[\mathrm{Xe}] .4 f^{4} .5 d^{10} .6 s^{2} .6 p^{2}$
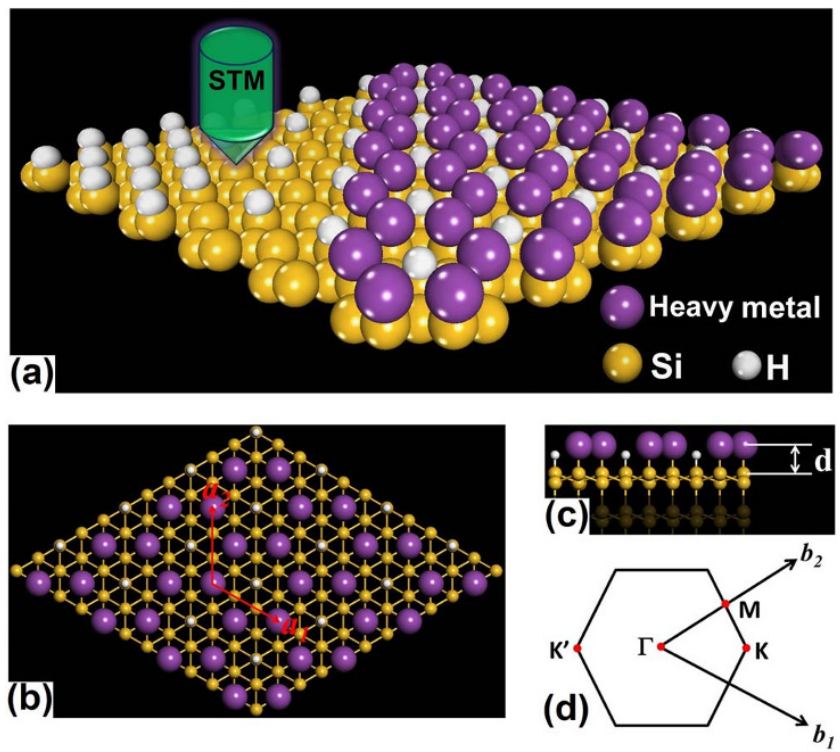

Figure 1 | A two-step approach to fabricate 2D TI by deposition of heavy metal atoms on a patterned $\mathrm{H}-\mathrm{Si}$ (111) surface. (a) Schematic view. (b, c) The top and side view of the proposed structure, with the surface unit cell vector $\left(a_{1}, a_{2}\right)$ indicated in (b) and the adsorption length $d$ in (c). (d) The first surface Brillouin zone. for $\mathrm{Pb}$ and $[\mathrm{Xe}] .4 f^{4} .5 d^{10} .6 s^{2} .6 p^{3}$ for Bi. With two electrons less in $\mathrm{Pb} @$ $\mathrm{H}-\mathrm{Si}(111)$ per unit cell, the lower Dirac band becomes almost unoccupied compared to that of Bi@H-Si(111).

Next, we include SOC in calculation, and the resulting band structures of $\mathrm{Bi}$ and $\mathrm{Pb} \mathrm{H}-\mathrm{Si}(111)$ are shown in Figs. 2(c) and (d), respectively. One sees that for $\mathrm{Bi} @ \mathrm{H}-\mathrm{Si}(111)$, two Dirac bands are split apart; one large energy gap of $\sim 0.7 \mathrm{eV}$ opens at $K$ point, and another gap of $\sim 0.5 \mathrm{eV}$ opens at $\Gamma$ point, which is the global gap. The $p_{\mathrm{y}}$ and the upper $p_{\mathrm{x}}$ bands are also separated by SOC, with an indirect gap $\sim 0.45 \mathrm{eV}$. We note that the spin degeneracy of these bands is lifted with most noticeable splitting at $K$ point, which is due to the Rashba effect ${ }^{35}$ induced by the broken spatial inversion symmetry. Although Rashba effect has shown to be detrimental to QSH phase ${ }^{19}$, in our systems such extrinsic spin splitting is relatively small compared to the intrinsic SOC induced band gap [see Fig. 2(c)], suggesting the QSH state is robust against Rashba effect in our surface systems. It should also be noted that the SOC strength of $\mathrm{Si}$ is orders of magnitude smaller that $\mathrm{Bi}$, making the $\mathrm{SOC}$ of $\mathrm{Bi}$ a decisive factor in opening the energy gap. Similarly, the SOC opens a gap at $K$ point for Pb@H-Si(111), with the upper branch of Dirac bands moving completely into the Si conduction band, as shown in Fig. 2(d). Nevertheless, the energy splitting between the two Dirac bands is found to be around $0.65 \mathrm{eV}$ at $K$ point, and an effective SOC gap of $\sim 0.54 \mathrm{eV}$ could be counted by the energy difference between the $\mathrm{Si}$ conduction band minimum and the top of the $\mathrm{Pb} p_{\mathrm{x}}$ band. However, to truly make the $\mathrm{Pb} \mathrm{H}-\mathrm{Si}(111)$ a 2D TI, n-type doping is needed to shift the Fermi level into the SOC gap, such as by $\mathrm{Si}$ substrate doping or electric gating.

The SOC-induced gap opening at the Dirac point in Bi@H-Si(111) and $\mathrm{Pb} @ \mathrm{H}-\mathrm{Si}(111)$ indicates possible existence of 2D TI state. To check this, we calculated the topological edge states of Bi@H-Si(111) by the Wannier90 package ${ }^{36}$. Using DFT bands as input, we construct the maximally localized Wannier functions and fit a tight-binding Hamiltonian with these functions. Figure 3(a) shows the DFT and fitted band structures, which are in very good agreement. Then, the edge Green's function of a semi-infinite Bi@H-Si(111) is constructed and the local density of state (DOS) of Bi zigzag edge is calculated, as shown in Fig. 3(b). Clearly, one sees gapless edge states that connect the upper and lower band edge of the bulk gap, forming a 1D Dirac cone at the center of Brillouin zone ( $\Gamma$ point). This indicates that the $\mathrm{Bi@H}-\mathrm{Si}(111)$ is a 2D TI with a large gap of $\sim 0.5 \mathrm{eV}$.

To further confirm the above topological edge-state results, we also calculated $Z_{2}$ topology number. As the spatial inversion symmetry is broken in these systems, we used the method developed by Xiao et al. ${ }^{22,37}$. In this method, $\mathrm{Z}_{2}$ is calculated by considering the Berry gauge potential and Berry curvature associated with the Bloch wave functions, which does not require any specific point-group symmetry. Indeed, we found that $\mathrm{Z}_{2}=1$ for Bi@H-Si(111) (Fig. S1 in Supplementary Information), confirming the existence of QSH state in this surface. Assuming a shift of Fermi level above the lower branch of Dirac band, we also found $\mathrm{Z}_{2}=1$ for Pb@H-Si(111) (Fig. S2).

The physical origin of QSH state in Bi@H-Si(111) and Pb@H$\mathrm{Si}(111)$ can be understood by a substrate orbital filtering effect as discussed recently in a related system of $\mathrm{Bi}$ on halogenated Si surfaces ${ }^{27}$. Figure 3(c) shows the partial density of states (DOS) of Bi@H$\mathrm{Si}(111)$ around the Fermi level. It is seen that the $p_{\mathrm{z}}$ orbital of $\mathrm{Bi}$ hybridizes strongly with the dangling bond of the exposed surface $\mathrm{Si}$ atom overlapping in the same energy range, which effectively removes the $p_{\mathrm{z}}$ bands away from the Fermi level, leaving only the $p_{\mathrm{x}}$ and $p_{\mathrm{y}}$ orbitals. We also analyzed the charge density redistribution [see upper panel of Fig. 3(d)], which clearly shows that charge redistribution induced by Si surface mainly happens to the $p_{z}$ orbital of $\mathrm{Bi}$, in a similar way to the saturation of $\mathrm{Bi} p_{z}$ orbital by using hydrogen [lower panel of Fig. 3(d)]. It has been shown that the free-standing planar hexagonal lattice of $\mathrm{Bi}$ is a topologically trivial insulator with 

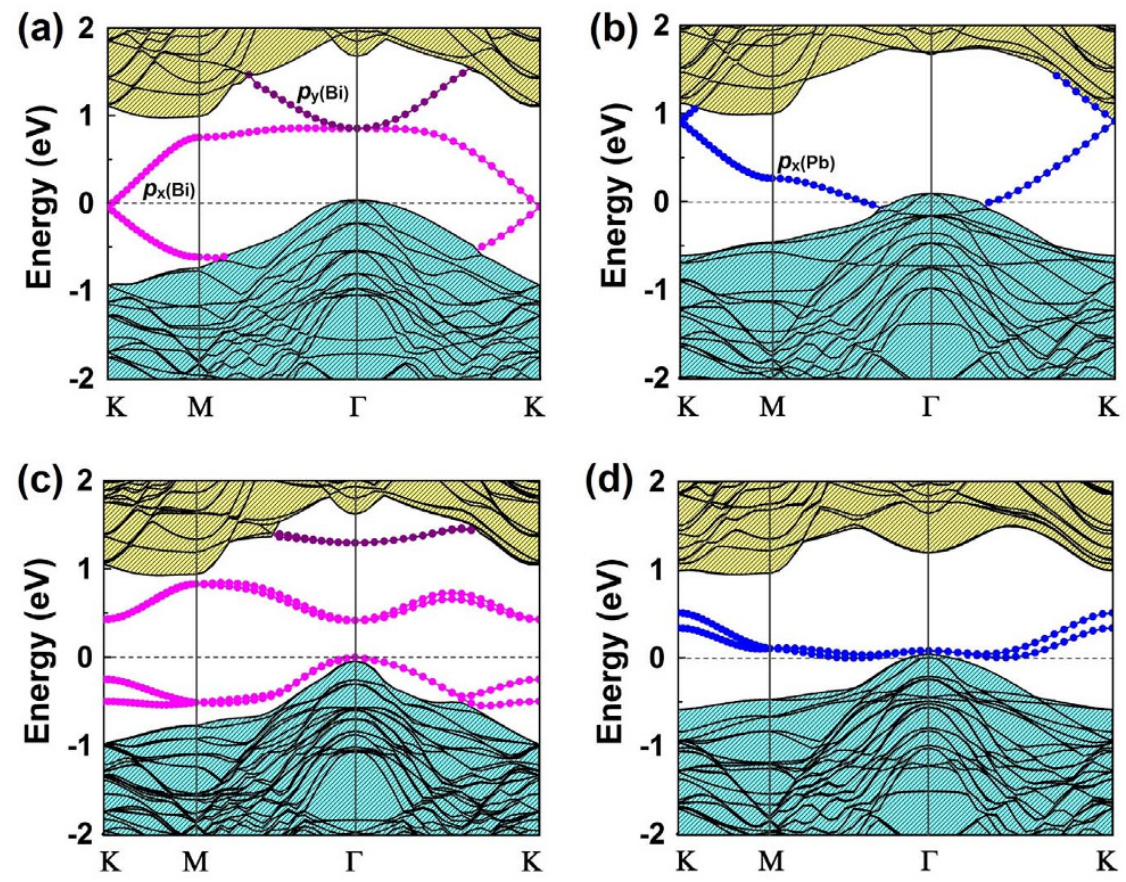

Figure $2 \mid$ Band structures of Bi and Pb@H-Si(11). (a-b) Without SOC. The Fermi level is set at zero. The green (yellow) shaded area represents the valence bands (conduction bands) of Si. Band compositions around Fermi level are also indicated. (c-d) Same as (a-b) with SOC.
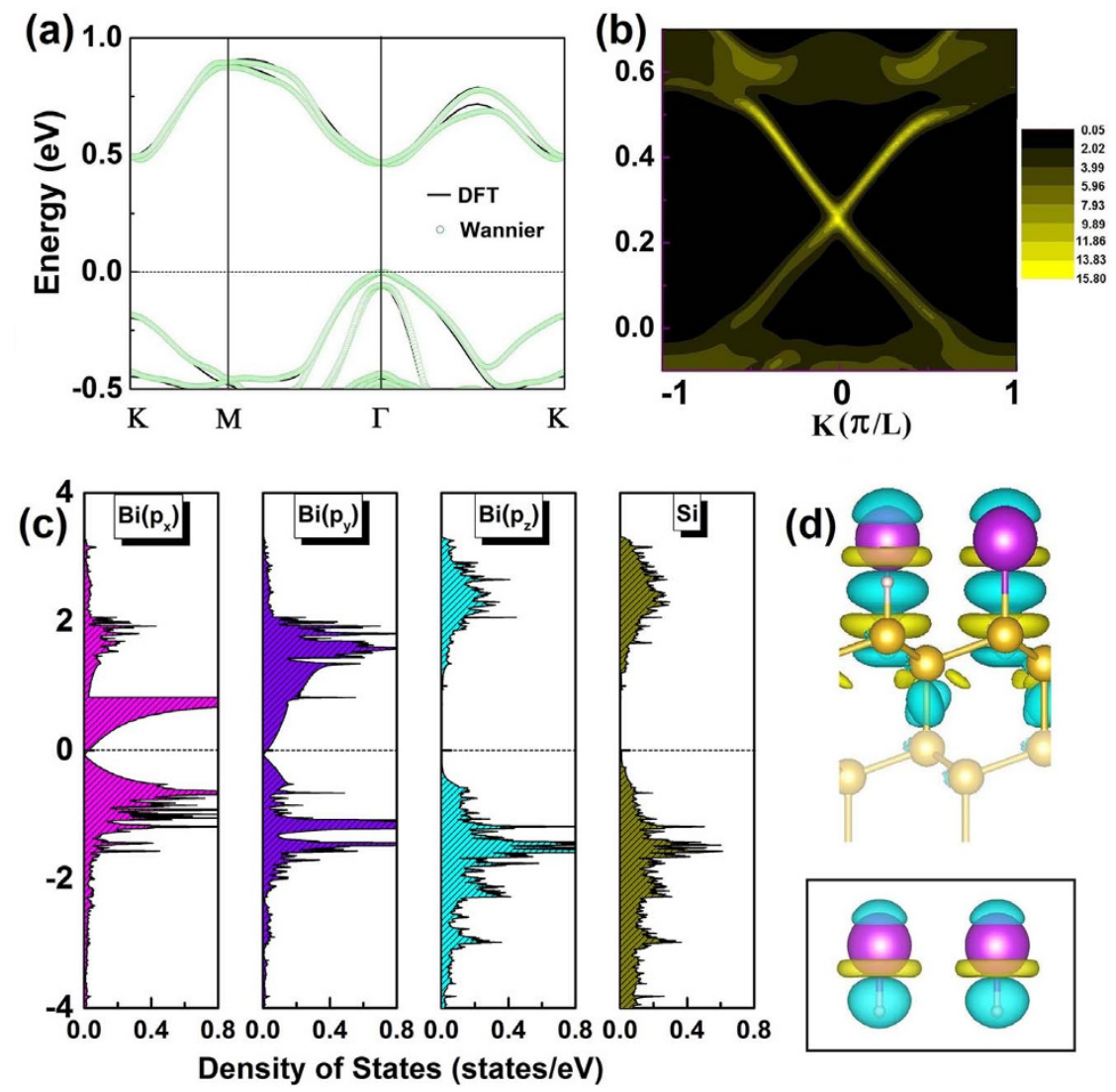

Figure 3 Electronic structures of Bi@H-Si(111) and its edge state. (a) Comparison of band structures for Bi@H-Si(111) calculated by DFT (black lines) and Wannier function method (green circles). (b) The Dirac edge states within the SOC-induced band gap. Scale bar is indicated on the right.

(c) The partial DOS projected onto $p_{\mathrm{x}}, p_{\mathrm{y}}$, and $p_{\mathrm{z}}$ orbitals of $\mathrm{Bi}$, and the total DOS of neighboring Si atoms. (d) Top: The charge density redistribution induced by metal atom surface adsorption for $\mathrm{Bi} @ \mathrm{H}-\mathrm{Si}(111)$ (isovalue $=0.02 \mathrm{e} / \mathrm{\AA}^{3}$ ), illustrating saturation of Bi $p_{\mathrm{z}}$ orbital. Bottom: Same as Top for the $\mathrm{H}$-saturated freestanding planar Bi lattice. 
$Z_{2}=0$ (see Fig. S3). When it is placed onto the H-Si(111) surface or adsorbed with $\mathrm{H}$, it becomes topologically nontrivial (Figs. S1 and S4). This originates from the intriguing orbital filtering effect imposed by the substrate or $\mathrm{H}$ saturation, which selectively remove the $p_{z}$ orbitals from the Bi lattice to realize the large-gap QSH phase.

Specifically, we can describe the Bi@H-Si(111) using a simplified $\left(p_{\mathrm{x}}, p_{\mathrm{y}}\right)$ four-band model Hamiltonian in a hexagonal lattice $\mathrm{as}^{24,38}$,

$\hat{H}$ eff $=\left(\begin{array}{cccc}\varepsilon_{0} & 0 & S_{x x} & S_{x y} \\ 0 & \varepsilon_{0} & S_{x y} & S_{y y} \\ S_{x x}^{*} & S_{x y}^{*} & \varepsilon_{0} & 0 \\ S_{x y}^{*} & S_{y y}^{*} & 0 & \varepsilon_{0}\end{array}\right)+\sigma_{Z} \lambda_{s o}\left(\begin{array}{cccc}0 & -i & 0 & 0 \\ i & 0 & 0 & 0 \\ 0 & 0 & 0 & -i \\ 0 & 0 & i & 0\end{array}\right)$,

in which $S_{x x}=V_{p p \sigma}+\left(\frac{1}{4} V_{p p \sigma}+\frac{3}{4} V_{p p \pi}\right) *\left(e^{i k a 1}+e^{i k(a 1+a 2)}\right), S_{x y}=\frac{3}{4}$ $\left(V_{p p \sigma}+V_{p p \pi}\right) *\left(e^{i k a 1}+e^{i k(a 1+a 2)}\right), \quad S_{y y}=V_{p p \pi}+\left(\frac{3}{4} V_{p p \sigma}+\frac{1}{4} V_{p p \pi}\right) *$ $\left(e^{i k a 1}+e^{i k(a 1+a 2)}\right), a_{1}, a_{2}$ is the lattice vector, $V_{p p \sigma}\left(V_{p p \pi}\right)$ is the Slater-Koster parameter ${ }^{39}$, and $\sigma_{Z}= \pm 1$ is the spin eigenvalue.

Diagonalization of Eq. (1) in reciprocal space gives the band structures shown in Fig. 4, which shows typical four bands as a function of SOC strength. One sees that without SOC, this Hamiltonian produces two flat bands and two Dirac bands with a Dirac point formed at $K$ point and two quadratic points at $\Gamma$ point [Fig. 4(a)]. Inclusion of a small SOC $(\lambda=0.2 t)$ opens one energy gap $\left(\Delta \mathrm{E}_{1}\right)$ at $K$ point and two energy gaps $\left(\Delta \mathrm{E}_{2}\right)$ at $\Gamma$ point [Fig. $4(\mathrm{~b})$ ], with both gaps topologically nontrivial ${ }^{24}$. With the increasing $\mathrm{SOC}$ strength, both $\Delta \mathrm{E}_{1}$ and $\Delta \mathrm{E}_{2}$ increase [Fig. 4(c)], which eventually leads to the formation of a different energy gap $\left(\Delta \mathrm{E}_{3}\right)$ between the upper and lower Dirac bands at $\Gamma$ point when $\Delta \mathrm{E}_{3}$ becomes smaller than both $\Delta \mathrm{E}_{1}$ and $\Delta \mathrm{E}_{2}$ [Fig. 4(d)]. As such, for sufficiently large SOC, $\Delta \mathrm{E}_{3}$ replaces $\Delta \mathrm{E}_{1}$ to be the global gap, and correspondingly the global gap shifts from $K$ to $\Gamma$ point. Further increase of SOC will tend to decrease $\Delta \mathrm{E}_{3}$, indicating that for sufficiently large SOC the band gap decreases with increasing SOC.

Such interesting phenomenon has also been confirmed by the DFT results. By comparing $\mathrm{Bi} @ \mathrm{H}-\mathrm{Si}(111)$ and $\mathrm{Pb} @ \mathrm{H}-\mathrm{Si}(111)$, we see that given the correct Fermi energy, the global gap is located at $\Gamma$ point for Bi@H-Si(111) [Fig. 2(c)], but at $K$ point for $\mathrm{Pb} @ \mathrm{H}-$ $\mathrm{Si}(111)$ [Fig. 2(d)]. This is because the SOC strength in $p$ orbital of $\mathrm{Pb}(\sim 0.91 \mathrm{eV})$ is smaller than that of $\mathrm{Bi}(\sim 1.25 \mathrm{eV})^{40}$. Meanwhile, the energy gap between the two $p_{\mathrm{y}}$ Dirac bands induced by SOC is actually larger for $\mathrm{Pb} @ \mathrm{H}-\mathrm{Si}(111)(0.65 \mathrm{eV})$ than that of $\mathrm{Bi} @ \mathrm{H}-$ $\mathrm{Si}(111)(0.5 \mathrm{eV})$, suggesting that $\mathrm{Pb}$ may be a better choice to achieve large-gap QSH states on the substrate. This is in sharp contrast with the Kane-Mele model in graphene, for which an energy gap is opened at Dirac point that is in proportion to the strength of $\mathrm{SOC}^{19}$.

Besides $\mathrm{Bi}$ and $\mathrm{Pb}$, we have also conducted calculations of other heavy elements adsorption on the Si surface, including Sb, Sn, Tl, In and Ga. It is found that $\mathrm{Sb}$ and $\mathrm{Sn}$ have a similar band structure with $\mathrm{Bi}$ and $\mathrm{Sn}$, respectively (see Fig. S5 in Supplementary Information), but with a smaller energy gap resulting from their similar valence electron configurations but weaker SOC. Band structures of Tl, In and Ga@H-Si(111) are a bit different. As shown in Fig. 5, the Fermi level now sits further below the lower dispersive band that is mainly made of the heavy atom $p_{y}$ orbital. This is due to the one (two) less valence electron compared to the $\mathrm{Pb}(\mathrm{Bi})$ group, i.e., $[\mathrm{Xe}] .4 f^{4} \cdot 5 d^{10} \cdot 6 s^{2} .6 p^{1}$ for Tl. Clearly, one sees that from Ga to Tl, the SOC gap between the lower Dirac band and dispersive band increases dramatically, from around $0.1 \mathrm{eV}$ (for $\mathrm{Ga}$ ) to $0.5 \mathrm{eV}$ for $(\mathrm{Tl})$, confirming the dependence of energy gap $\left(\Delta \mathrm{E}_{2}\right)$ on SOC as demonstrated in Fig. 4.

In summary, we demonstrate the possibility of 'controlled' growth of large-gap topological quantum phases on conventional substrate surfaces such as the important Si surface by a unique approach of substrate orbital filtering process combined with a proper choice of SOC. Its underlying physical principles are general, applicable to
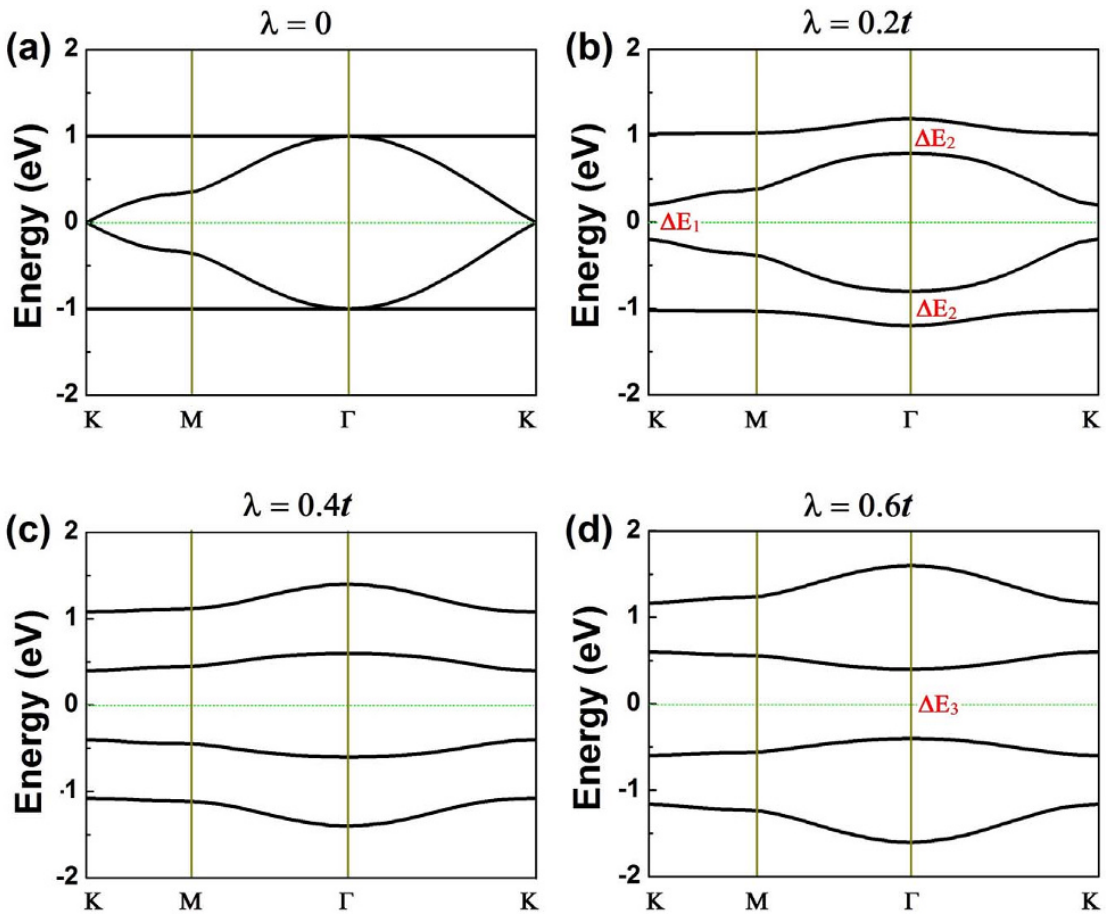

Figure $4 \mid$ Energy bands resulting from the four-band model [Eq. (1)] as a function of SOC strength ( $\lambda$ ) scaled by $t$ ( $t$ is the coupling strength between neighboring $p_{x}$ and $p_{y}$ orbitals). Fermi energy is set to zero. The SOC induced energy gaps $\left(\Delta \mathrm{E}_{1}, \Delta \mathrm{E}_{2}\right.$ and $\left.\Delta \mathrm{E}_{3}\right)$ are indicated. The global gap transition from $K$ point to $\Gamma$ point driven by SOC can be clearly seen. 

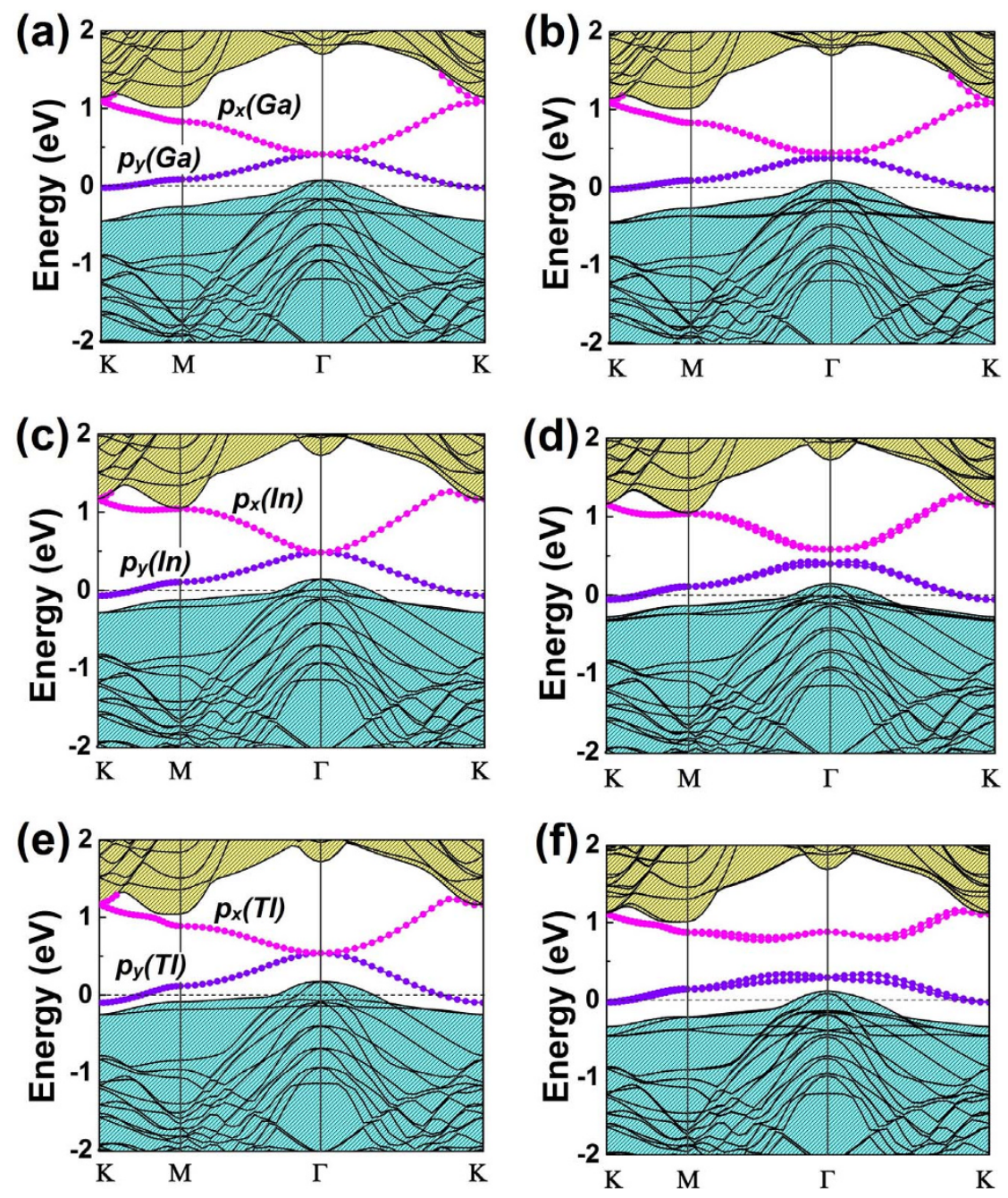

Figure $5 \mid$ Band structures of Ga, In, Tl@H-Si(111). (a-b) Band structures of Ga@H-Si(11) without and with SOC, respectively. The Fermi level is set to zero. Band compositions around Fermi level are indicated. (c-d) Same as (a-b) for In@H-Si(111). (e-f) Same as (a-b) for Tl@H-Si(111).

deposition of different metal atoms on different substrates ${ }^{11,27}$. It opens up a new and exciting avenue for future design and fabrication of room temperature topological surface/interface states based on current available epitaxial growth and semiconductor technology, where the metal overlayer is atomically bonded but electronically isolated from the underneath semiconductor substrate ${ }^{27}$.

\section{Methods}

Our electronic structure calculations based on density functional theory were performed by using a plane wave basis set ${ }^{41}$ and the projector-augmented wave method ${ }^{42}$ as implemented in the VASP code ${ }^{43}$. The exchange-correlation functional was treated with the generalized gradient approximation in Perdew-Burke-Ernzerhof format ${ }^{44}$. Calculations of $Z_{2}$ triviality were carried out by using the full-potential linearized augmented plane-wave method implemented in the WIEN2K package ${ }^{45}$. Details for models and computations ( $\mathrm{Z} 2$ invariant calculation results, and band structures of $\mathrm{Sb}$ and $\mathrm{Sn} @ \mathrm{H}-\mathrm{Si}(111))$ are presented in Supplementary Information.

1. Moore, J. E. The birth of topological insulators. Nature 464, 194-198 (2010).

2. Hasan, M. Z. \& Kane, C. L. Colloquium: topological insulators. Rev. Mod. Phys. 82, 3045-3067 (2010).

3. Qi, X.-L. \& Zhang, S.-C. Topological insulators and superconductors. Rev. Mod. Phys. 83, 1057-1110 (2011).

4. Bernevig, B. A. \& Zhang, S.-C. Quantum spin hall effect. Phys. Rev. Lett. 96, 106802 (2006).

5. Xu, C. \& Moore, J. E. Stability of the quantum spin Hall effect: Effects of interactions, disorder, and $\mathrm{Z}_{2}$ topology. Phys. Rev. B 73, 045322 (2006).

6. Xia, Y. et al. Observation of a large-gap topological-insulator class with a single Dirac cone on the surface. Nat. Phys. 5, 398-402 (2009).
7. Yang, F. et al. Spatial and energy distribution of topological edge states in single Bi(111) bilayer. Phys. Rev. Lett. 109, 016801 (2012).

8. Hirahara, T. et al. Atomic and electronic structure of ultrathin $\mathrm{Bi}(111)$ films grown on $\mathrm{Bi}_{2} \mathrm{Te}_{3}(111)$ substrate: Evidence for a strain-induced topological phase transition. Phys. Rev. Lett. 109, 227401 (2012).

9. Miao, L. et al. Quasiparticle dynamics in reshaped helical Dirac cone of topological insulators. Proc. Natl. Acad. Sci. U.S.A. 110, 2758 (2013).

10. Wang, Z. F. et al. Creation of helical Dirac fermions by interfacing two gapped systems of ordinary fermions. Nat. Commun. 4, 1384 (2013).

11. Zhou, M., Liu, Z., Ming, W., Wang, Z. F. \& Liu, F. sd ${ }^{2}$ Graphene: Kagome band in a hexagonal lattice. arXiv: 1411.0786 .

12. König, M. et al. Quantum spin Hall insulator state in HgTe quantum wells. Science 318, 766-770 (2007).

13. Chen, Y. L. et al. Experimental realization of a three-dimensional topological insulator, $\mathrm{Bi}_{2} \mathrm{Te}_{3}$. Science 325, 178-181 (2009).

14. Murakami, S. Quantum spin Hall effect and enhanced magnetic response by spinorbit coupling. Phys. Rev. Lett. 97, 236805 (2006).

15. Wada, M., Murakami, S., Freimuth, F. \& Bihlmayer, G. Localized edge states in two-dimensional topological insulators: Ultrathin Bi films. Phys. Rev. B $\mathbf{8 3}$, 121310 (2011).

16. Liu, Z. et al. Stable nontrivial $Z_{2}$ topology in ultrathin Bi (111) films: a firstprinciples study. Phys. Rev. Lett. 107, 136805 (2011).

17. Zhang, P. F., Liu, Z., Duan, W., Liu, F. \& Wu, J. Topological and electronic transitions in a $\mathrm{Sb}(111)$ nanofilm: The interplay between quantum confinement and surface effect. Phys. Rev. B 85, 201410 (2012).

18. Xu, Y. et al. Large-gap quantum spin Hall insulators in Tin Films. Phys. Rev. Lett. 111, 136804 (2013)

19. Kane, C. L. \& Mele, E. J. Quantum spin Hall effect in graphene. Phys. Rev. Lett. 95 , 226801 (2005)

20. Weeks, C., Hu, J., Alicea, J., Franz, M. \& W, R. Engineering a robust quantum spin Hall state in graphene via adatom deposition. Phys. Rev. X 1, 021001 (2011). 
21. Hu, J., Alicea, J., W, R. \& Franz, M. Giant topological insulator gap in graphene with 5d adatoms. Phys. Rev. Lett. 109, 266801 (2012).

22. Liu, C. C., Feng, W. \& Yao, Y. Quantum spin Hall effect in silicene and twodimensional germanium. Phys. Rev. Lett. 107, 076802 (2011).

23. Wang, Z. F., Liu, Z. \& Liu, F. Organic topological insulators in organometallic lattices. Nat. Commun. 4, 1471 (2013).

24. Liu, Z., Wang, Z. F., Mei, J. W., Wu, Y. S. \& Liu, F. Flat Chern Band in a TwoDimensional Organometallic Framework. Phys. Rev. Lett. 110, 106804 (2013).

25. Wang, Z. F., Su, N. \& Liu, F. Prediction of a Two-Dimensional Organic Topological Insulator. Nano Lett. 13, 2842-2845 (2013).

26. Wang, Z. F., Liu, Z. \& Liu, F. Quantum anomalous Hall effect in 2D organic topological insulator. Phys. Rev. Lett. 110, 196801 (2013).

27. Zhou, M., Ming, W., Liu, Z., Wang, Z. F., Li, P. \& Liu, F. Epitaxial growth of largegap quantum spin Hall insulator on semiconductor surface. Proc. Natl. Acad. Sci. U.S.A. 111, 14378 (2014)

28. Higashi, G. S., Chabal, Y. J., Trucks, G. W. \& Raghavachari, K. Ideal hydrogen termination of the Si-(111) surface. Appl. Phys. Lett. 56, 656-658 (1990).

29. Tada, H., Kawaguchi, T. \& Koma, A. Epitaxial growth of vanadyl-phthalocyanine ultrathin films on hydrogen-terminated Si(111) surfaces. Appl. Phys. Lett. 61, 2021-2023 (1992).

30. Shen, T.-C. et al. Atomic-scale desorption through electronic and vibrational excitation mechanisms. Science 268, 1590-1592 (1995).

31. Schofield, S. R. et al. Quantum engineering at the silicon surface using dangling bonds. Nat. Commun. 4, 1649 (2013).

32. Gomes, K. K., Mar, W., Ko, W., Guinea, F. \& Manoharan, H. C. Designer Dirac fermions and topological phases in molecular graphene. Nature 483, 306-310 (2012).

33. Polini, M., Guinea, F., Lewenstein, M., Manoharan, H. C. \& Pellegrini, V. Artificial honeycomb lattices for electrons, atoms and photons. Nat. Nanotechnol. 8, 625-633 (2013).

34. Michalak, D. J. et al. Nanopatterning $\mathrm{Si}(111)$ surfaces as a selective surfacechemistry route. Nat. Mater. 9, 266-271 (2010).

35. Bychkov, Y. A. \& Rashba, E. I. Properties of a 2D electron gas with lifted spectral degeneracy. JETP Lett. 39, 78 (1984).

36. Mostofi, A. A. et al. Wannier90: a tool for obtaining maximally-localized wannier functions. Comput. Phys. Commun. 178, 685-699 (2008).

37. Feng, W. X., Wen, J., Zhou, J.-J., Xiao, D. \& Yao, Y. G. First-principles calculation of $Z_{2}$ topological invariants within the FP-LAPW formalism. Comput. Phys. Commun. 183, 1849-1859 (2012).

38. Wu, C. \& Das Sarma, S. $p_{\mathrm{x}, \mathrm{y}}$-orbital counterpart of graphene: Cold atoms in the honeycomb optical lattice. Phys. Rev. B 77, 235107 (2008)

39. Slater, J. C. \& Koster, G. F. Simplified LCAO method for the periodic potential problem. Phys. Rev. 94, 1498-1524 (1954).
40. Bian, G., Wang, X., Miller, T. \& Chiang, T.-C. Origin of giant Rashba spin splitting in Bi/Ag surface alloys. Phys. Rev B 88, 085427 (2013).

41. Blöchl, P. E. Projector augmented-wave method. Phys. Rev. B 50, 17953 (1994).

42. Kresse, G. \& Joubert, D. From ultrasoft pseudopotentials to the projector augmented-wave method. Phys. Rev. B 59, 1758 (1999).

43. Kresse, G. \& Hafner, J. Ab initio molecular dynamics for liquid metals. Phys. Rev. B 47, 558 (1993).

44. Perdew, J. P., Burke, K. \& Ernzerhof, M. Generalized Gradient Approximation Made Simple. Phys. Rev. Lett. 77, 3865 (1996).

45. Blaha, P., Schwarz, K., Madsen, G. K. H., Kvasnicka, D. \& Luitz, J. WIEN2K: An Augmented Plane Wave and Local Orbitals Program for Calculating Crystal Properties (Vienna University of Technology, Vienna, Austria, 2001).

\section{Acknowledgments}

This research was supported by DOE (Grant No: DEFG02-04ER46148); Z. F. Wang and W. Ming additionally thank support from NSF-MRSEC (Grant No. DMR-1121252). We thank NERSC and the CHPC at University of Utah for providing the computing resources.

\section{Author contributions}

M.Z. carried out the theoretical calculations with the assistance of W.M.M., Z.L., Z.F.W. and Y.G.Y.; F.L. guided the overall project. M.Z. and F.L. wrote the manuscript

\section{Additional information}

Supplementary information accompanies this paper at http://www.nature.com/ scientificreports

Competing financial interests: The authors declare no competing financial interests How to cite this article: Zhou, M. et al. Formation of quantum spin Hall state on Si surface and energy gap scaling with strength of spin orbit coupling. Sci. Rep. 4, 7102; DOI:10.1038/ srep07102 (2014).

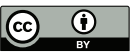

This work is licensed under a Creative Commons Attribution 4.0 International License. The images or other third party material in this article are included in the article's Creative Commons license, unless indicated otherwise in the credit line; if the material is not included under the Creative Commons license, users will need to obtain permission from the license holder in order to reproduce the material. To view a copy of this license, visit http://creativecommons.org/licenses/by/4.0/ 